\title{
Clinical, surgical and postoperative outcome analysis of tumors occupying both posterior and middle cranial fossa
}

\author{
Rahul Singh $M S^{1}$ (iD), Ravi Shankar Prasad $M C h^{2}$ iD, Ramit Chandra Singh $M S^{3}$ (D), Kulwant Singh $M C h^{4}$ (D), \\ Anurag Sahu $\mathrm{MCh}^{5}$ iD \\ 1,2,3,4,5 Department of Neurosurgery, Institute of Medical Sciences- Banaras Hindu University, Varanasi, Uttar Pradesh, India \\ Date of submission: $7^{\text {th }}$ January 2021 \\ Date of acceptance: $7^{\text {th }}$ May 2021 \\ Date of publication: $1^{\text {st }}$ June 2021
}

\begin{abstract}
Introduction: This study aims to analyse clinical, surgical and postoperative outcome perspectives of tumors occupying both middle and posterior cranial fossa.

Methods and Materials: This retrospective cohort study includes 23 patients operated for tumors involving both middle and posterior cranial fossa in our department between August 2016 and August 2020. Each patient was evaluated for age, sex, co-morbidities, tumour histopathology, clinical presentation, radiological characteristics, surgical and outcome characteristics. Unpaired t- test and chi-square tests were used for statistical analysis. $\mathrm{P}<0.05$ was considered statistically significant.

Results: The mean age was 46 years (range $40-57$ years), with the majority of patients being female (56.5\%). Most tumors $(65.3 \%)$ were trigeminal schwannoma, with the remaining being vestibular schwannoma $(21.7 \%)$, facial schwannoma (8.7\%) and epidermoid (4.3\%). The most commonly used surgical approach was the retrosigmoid approach (30.4\%) and combined approach (30.4\%). Gross total resection (GTR) was done in 14 patients while subtotal resection (STR) was done in 9 patients. Subtotal resection was significantly associated $(\mathrm{p}<0.05)$ with persisting cranial nerve deficit and tumour progression. Midbrain compression, internal carotid artery and cavernous sinus involvement were significantly $(\mathrm{p}<0.05)$ associated with subtotal resection.

Conclusions: Among bicompartmental tumors, trigeminal schwannoma is more common. Combined approach for such tumour is required in tumours having significant size in both middle cranial fossa and posterior cranial fossa. The intent of surgery is to achieve a gross total resection but adhesions and involvement of critical brain structures results in subtotal resection.
\end{abstract}

Key words: Epidermoid, Middle cranial fossa, Posterior cranial fossa, Trigeminal Schwannoma, Vestibular Schwannoma.

\section{Introduction}

Access this article online

Website: https://www.nepjol.info/index.php/NJN

DOI: https://doi.org/10.3126/njn.v18i2.33783

HOW TO CITE

Singh R, Prasad RS, Singh RC, Singh K, Sahu A. Cinıcaı, surgical and postoperative outcome analysis of tumors occupying both posterior and middle cranial fossa. NJNS. 2021;18(2):36-44.

Address for correspondence:

Dr Ravi Shankar Prasad

Associate Professor and Head

Department of Neurosurgery

Institute of Medical Sciences- Banaras Hindu University,

Varanasi, Uttar Pradesh, India.

E-mail: rsprasadbhuhodneurosurgery@gmail.com

Phone: +91 9452345652

Copyright (C) 2021 Nepalese Society of Neurosurgeons (NESON)

ISSN: 1813-1948 (Print), 1813-1956 (Online)

This work is licensed under a Creative Commons Attribution-Non Commercial 4.0 International License.
Tumours extending in both posterior and middle cranial fossa are challenging entities in neurosurgical perspective. Many vital structures and cranial nerves are clustered around the region of cerebellopontine angle and in midbrain vicinity. Therefore complete surgical excision of such multicompartment tumours is difficult.

The differential diagnosis of these lesions includes trigeminal schwannoma, acoustic schwannoma, meningioma, ependymoma, metastasis, and chondrosarcoma. ${ }^{1}$ Rarely facial schwannoma and epidermoid may also present in similar manner., ${ }^{2,3}$

Surgical approach depends on predominant compartment viz. middle cranial fossa or posterior fossa involved by tumour. Combined approach may be needed in case of very large tumours involving both compartments. In this study we aim to analyse clinical, surgical and postoperative outcome perspectives of such bicompartmental tumours which will help in better categorization, prognostication and surgical planning of such tumours. 


\section{Methods and Materials}

This retrospective cohort study includes a cohort of 23 patients who underwent surgery for involving both middle and posterior cranial fossa in our department between 1 August 2016 and 31 August 2020.

This is a retrospective cohort study in two phases: a cross-sectional phase where the patients included in the sample were evaluated for the following described variables and a follow-up phase at hospital discharge, 1 month and 6 months respectively. Each patient was evaluated in term of age, sex, co morbidities, tumour histopathology, clinical presentation, radiological characteristics, surgical and outcome characteristics.

The inclusion criteria were all patients operated in elective settings in our department having preoperative imaging (MRI brain, CECT brain) suggestive of intracranial SOLs (space occupying lesions) involving both middle cranial fossa and posterior cranial fossa between 1 August 2016 and 31 August 2020. Patients were excluded if they had undergone previous surgical intervention for the same lesion or had received radiotherapy.

Data was collected retrospectively by analysing medical and surgical records of 23 operated patients having tumour involving both middle cranial fossa and posterior fossa compartments, submitted in medical record department of our hospital between 1 August 2016 and 31 August 2020. Data was collected from the medical and surgical records and included patient demographics, clinical findings, and duration of symptoms, surgical details, tumour characteristics, radiological data and treatment given. Persisting complications were considered as those complications that persisted for six months or more postoperatively.

\section{Surgical Treatment}

The clinical status and preoperative radiological imaging were used for determining the appropriate surgical approach. CT angiography was done preoperatively to define adjacent vascular structures and feeding vessels to the tumor. Based on surgeon preference and radiological diagnosis appropriate surgical approach was selected. Written and informed consent were taken before surgery. For trigeminal schwannomas; Dolenc's frontotemporal epidural, presigmoid transpetrosal-transtentorial and extended middle fossa rhomboid approaches were used. The extended middle fossa approach and Dolenc's frontotemporal epidural approaches were used in cases of small posterior fossa extension; this extension can be removed via expanded Meckel cave. Presigmoid transpetrosal-transtentorial approach was used when part of the tumor extending into middle cranial fossa was small and predominantly involved posterior cranial fossa. For large dumbbell tumors involving both compartments combined approach was used. For other bicompartmental tumors, we used retrosigmoid approach for tumors with predominant posterior compartment extension and extended middle cranial fossa approach for tumors with predominant middle cranial fossa extension. Combined approach viz. both extended middle cranial fossa and retrosigmoid approach was used in tumors with significant extension in both the compartments. Microsurgical techniques, intraoperative neuronavigation and neuromonitoring were utilised during surgery. For epidermoids, neuroendoscope was used to aid removal of tumors from crevices. Dural closure was done by interrupted Vicryl 4-0 sutures. In cases, where dural defect was large we used pericranial grafts or artificial dural grafts. Fibrin glue was used in cases of anticipated cerebrospinal fluid (CSF) leak after dural repair. We do not prefer preoperative lumbar drain placement for prevention of CSF leak at our centre.

Gross-total resection (GTR) is defined as the complete resection of the tumour on postoperative MRI or CT scan whereas Sub-total resection (STR) is defined as any residual tumour on postoperative MRI or CT scan. Recurrent lesion was evaluated using postoperative MRI and characterized as the radiologically progressive tumour. Additionally, imaging studies and operative reports were used to determine tumour extension into the IAC, jugular foramen, cavernous sinus and internal carotid artery.

\section{Statistical Analysis}

The data were summarized using medians and mean for continuous variables, and counts and percentages for categorical variables. Differences in continuous variables and categorical variables were evaluated using the unpaired t- test and chi-square test respectively. Values with $\mathrm{p}<0.05$ were considered statistically significant. Statistical tests were done using GraphPad Prism version 8.3.0 software.

\section{Result}

\section{Patient and Tumour Characteristics}

A total of 23 patients underwent surgical treatment for this dual compartment tumour during the 4-year period. The mean age was 46 years (range $40-57$ years), with the majority of patients being female $(56.5 \%)$. Hypertension $(21.7 \%)$ was the most common comorbidity found in these patients followed by diabetes mellitus $(17.4 \%)$, hypothyroidism $(8.7 \%)$, respiratory disorders (13\%), alcoholic intake (26.1\%) and smoking (34.8\%). Based on Tumour histopathology, intraoperative findings and MRI/ CT brain findings most tumors $(65.3 \%)$ were trigeminal schwannoma, with the remaining being Vestibular schwannoma (21.7\%), Facial schwannoma (8.7\%) and Epidermoid (4.3\%). The median tumour size (maximum length) was $6.8 \mathrm{~cm}$ (range 5.4-9.3 cm). 


\section{Singh et al}

\section{Clinical Presentation}

The median duration of symptoms was 11 months (range: 5 - 36 months). The most common presenting symptom was headache $(91.3 \%)$; this was followed by facial numbness/pain (78.3\%), ataxia (52.2\%), facial paresis/paralysis (43.5\%) and hearing loss (43.5\%). Other presenting symptoms included tinnitus, seizure, and double vision, diminution of vision, dysarthria, nasal regurgitation and nasal intonation of speech and lowerextremity weakness. Most common cranial nerve involved was CN V (73.9\%) followed by CN VII (43.5\%) and CN VIII (26.1\%). Other cranial nerves involved were CN II, $\mathrm{CN}$ IV and lower cranial nerves (CN IX, X). Cerebellar signs were present in $52.2 \%$ of patients.

\section{Radiological Presentation}

On analysing preoperative MRI and CT scans of the brain we found that hydrocephalus and petrous apex destruction was present in $26.1 \%$ of patients. Other significant findings included midbrain compression (21.7\%), cavernous sinus involvement (13\%), internal carotid artery involvement (13\%) and optic chiasma compression $(8.7 \%)$.

\section{Surgical Characteristics}

A number of different surgical approaches were used for the resection of these dual compartment tumours. The most commonly used approach for trigeminal schwannoma was Dolenc's frontotemporal epidural (40\%) followed by retrosigmoid approach $(26.7 \%)$, extended middle cranial fossa $(13.3 \%)$ and combined approach (20\%). Most common approach for vestibular schwannoma used was retrosigmoid $(60 \%)$ followed by extended middle cranial fossa $(20 \%)$ and combined (20\%) approach. For facial schwannoma and epidermoid, retrosigmoid approach was used. Gross total resection (GTR) was achieved in 60.9\% of patients. STR was done in 9 patients of trigeminal schwannoma and 1 patient of vestibular schwannoma.

Nine patients (39.1\%), 4 of vestibular schwannoma and 5 of trigeminal schwannoma underwent postoperative radiotherapy, all of whom received STR.

\section{Postoperative Outcomes}

New cranial nerve deficits occurred in $26 \%$ consisting of CN VII (21.7\%) and lower cranial nerves (4.3\%). Of these new cranial nerve deficits $13 \%$ persisted and $8.7 \%$ recovered later in the postoperative period. Persisting cranial nerve deficit including both pre-existing and new deficits occurred in $78.3 \%$ patients. Other complications included wound infection, CSF leaks, tracheostomy requirement, and aspiration pneumonitis due to involvement of lower cranial nerves requiring Ryle's tube placement and hematoma. Regional complication in the form of wound infection, CSF leak and hematoma occurred in a patient $(4.3 \%)$ operated for trigeminal schwannoma by combined approach. Systemic complication occurred in the form of tracheostomy requirement due to prolonged intubation, aspiration pneumonitis and cranial nerve deficits.

Systemic complications were more after resection of Trigeminal schwannoma (66.7\%) followed by Vestibular schwannoma $(60 \%)$. Regional complication in the form of wound infection and CSF leak was present in 1 patient of Vestibular schwannoma (20\%). New neurologic deficit viz. Cranial nerve deficits were more common in Vestibular schwannoma $(60 \%)$ followed by facial schwannoma (50\%). Gross total resection (GTR) was possible in $100 \%$ patients of facial schwannoma and epidermoid patients.

No significant difference was found between GTR and STR group with respect to new cranial nerve deficit, systemic and regional complications. STR was significantly associated $(p<0.05)$ with permanent cranial nerve deficit $(\mathrm{p}<0.0343)$ and tumour progression $(\mathrm{p}<$ 0.0001 ). At a mean follow-up duration of 9.2 months (range 0-24 months), nine patients (39.13\%) had tumour progression. All patients with tumour progression underwent STR during the primary procedure (Table 1).

Midbrain compression $(\mathrm{p}=0.0343)$, internal carotid artery $(p=0.0285)$ and cavernous sinus involvement $(p=0.0285)$ was significantly associated with STR (Table $1)$.

\begin{tabular}{|c|c|c|c|c|}
\hline S.No & Outcome & GTR $(n=14)$ & $\operatorname{STR}(n=9)$ & $\overline{P \text { value }}$ \\
\hline 1. & New cranial nerve deficit & $5(35.7 \%)$ & $1(11.1 \%)$ & 0.1897 \\
\hline 2. & Persisting cranial nerve deficit & $9(64.3 \%)$ & $9(100 \%)$ & 0.0343 \\
\hline 3. & Tumour progression & 0 & $9(100 \%)$ & $<0.0001$ \\
\hline 4. & Regional complications & $1(7.1 \%)$ & 0 & 0.4123 \\
\hline \multirow[t]{2}{*}{5.} & Systemic complications & $8(57.1 \%)$ & $5(55.5 \%)$ & 0.9403 \\
\hline & Radiological characteristics & GTR & STR & P value \\
\hline 1. & Midbrain compression $(n=5)$ & $1(20 \%)$ & $4(80 \%)$ & 0.0343 \\
\hline 2. & Internal carotid artery involvement $(n=3)$ & 0 & $3(100 \%)$ & 0.0285 \\
\hline 3. & Cavernous sinus involvement $(\mathrm{n}=3)$ & 0 & $3(100 \%)$ & 0.0285 \\
\hline 4. & Petrous apex destruction $(n=6)$ & $4(66.7 \%)$ & $2(33.3 \%)$ & 0.7350 \\
\hline 5. & Optic chiasma compression $(n=2)$ & $1(50 \%)$ & $1(50 \%)$ & 0.8028 \\
\hline 6. & Hydrocephalus $(n=6)$ & $4(66.7 \%)$ & $2(33.3 \%)$ & 0.7350 \\
\hline
\end{tabular}

Table 1: Postoperative outcome and resectability analysis 
Clinical, surgical and postoperative outcome analysis of bicompartmental intracranial tumours
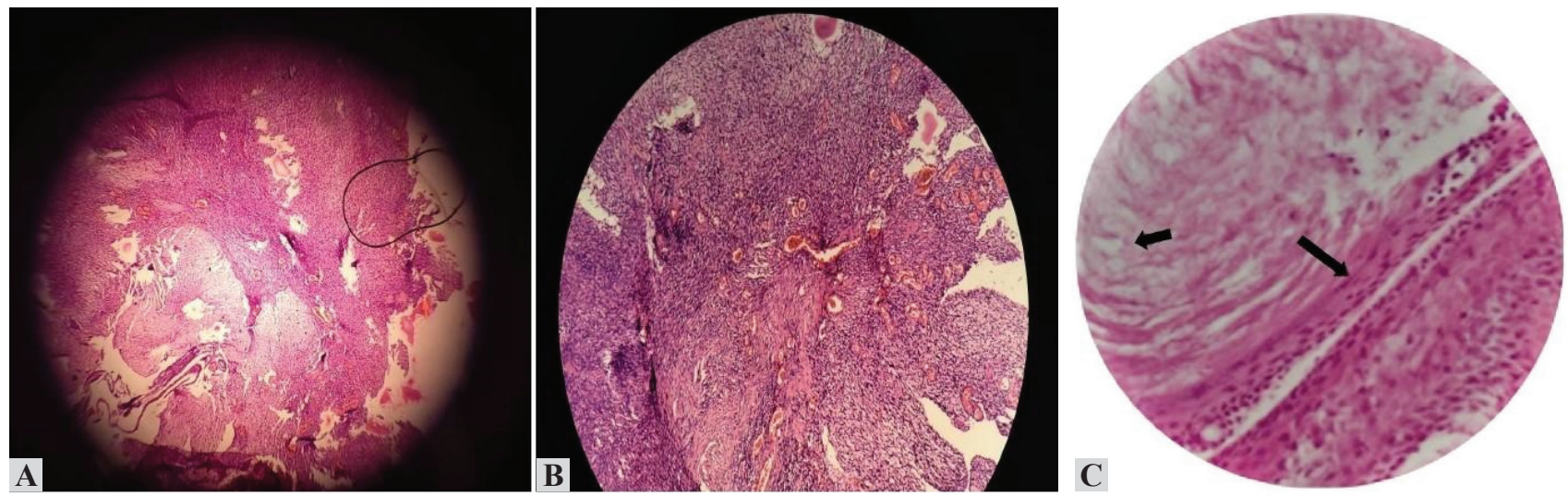

Figure 1: Histopathology in H \& E stain 10X magnification. Cranial nerve schwannoma (A) Antoni A and Antoni B areas; (B) Palisading pattern of schwannoma. Intracranial epidermoid (C) Small arrow, keratin flakes and large arrow, stratified squamous epithelium.
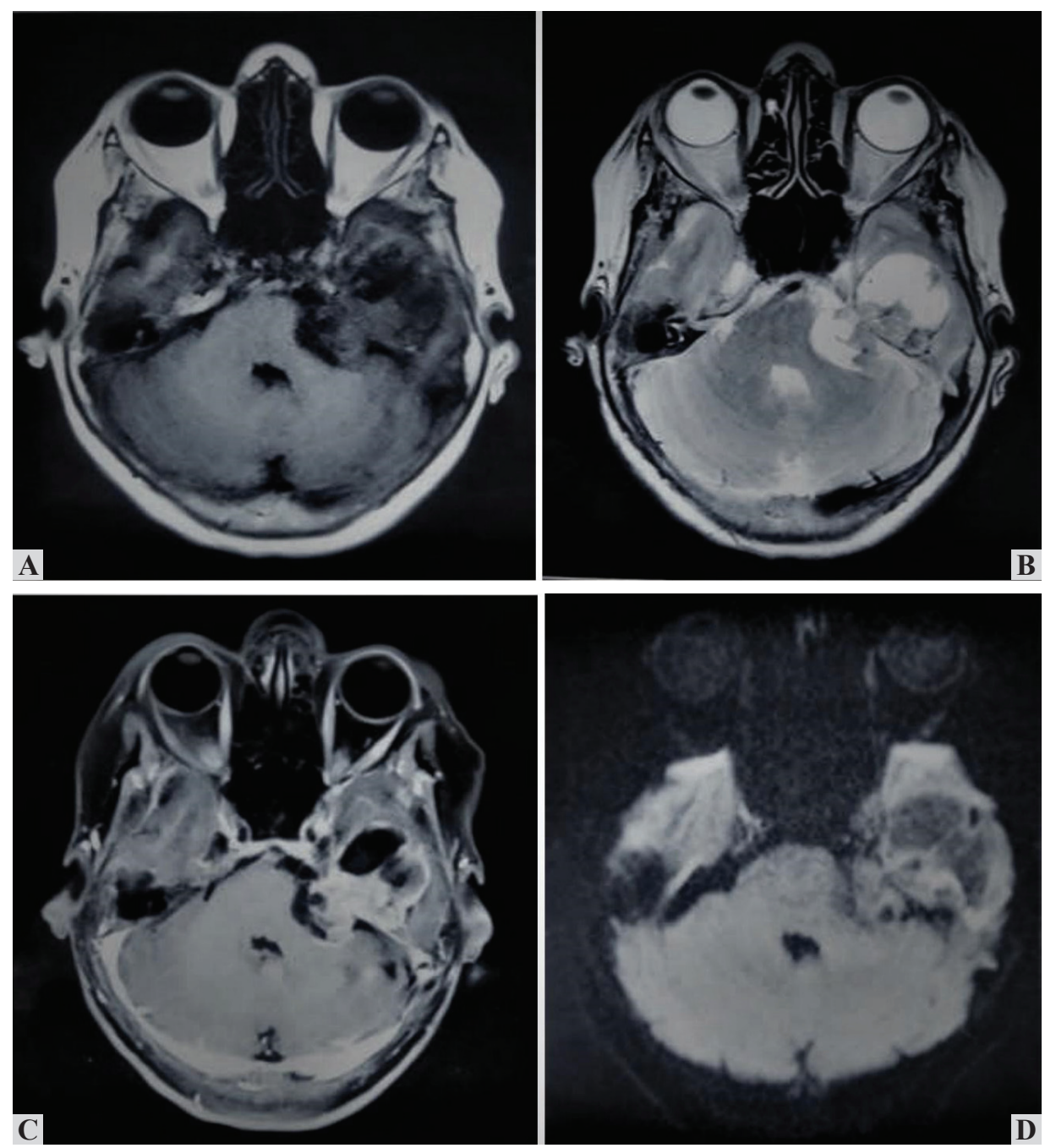

Figure 2: (A) axial T1 image showing a well-defined extra-axial hypointense cystic lesion in left cerebellopontine angle region causing mass effect over brainstem and cerebellum extending into the middle cranial fossa through destruction of petrous temporal bone suggestive of cystic vestibular schwannoma. (B) axial T2 image showing heterogenous hyperintense lesion. (C) axial FLAIR image showing no suppression with heterogenous hyperintense signal. (D) axial DWI image showing dark signals representing no diffusion restriction. 
Singh et al
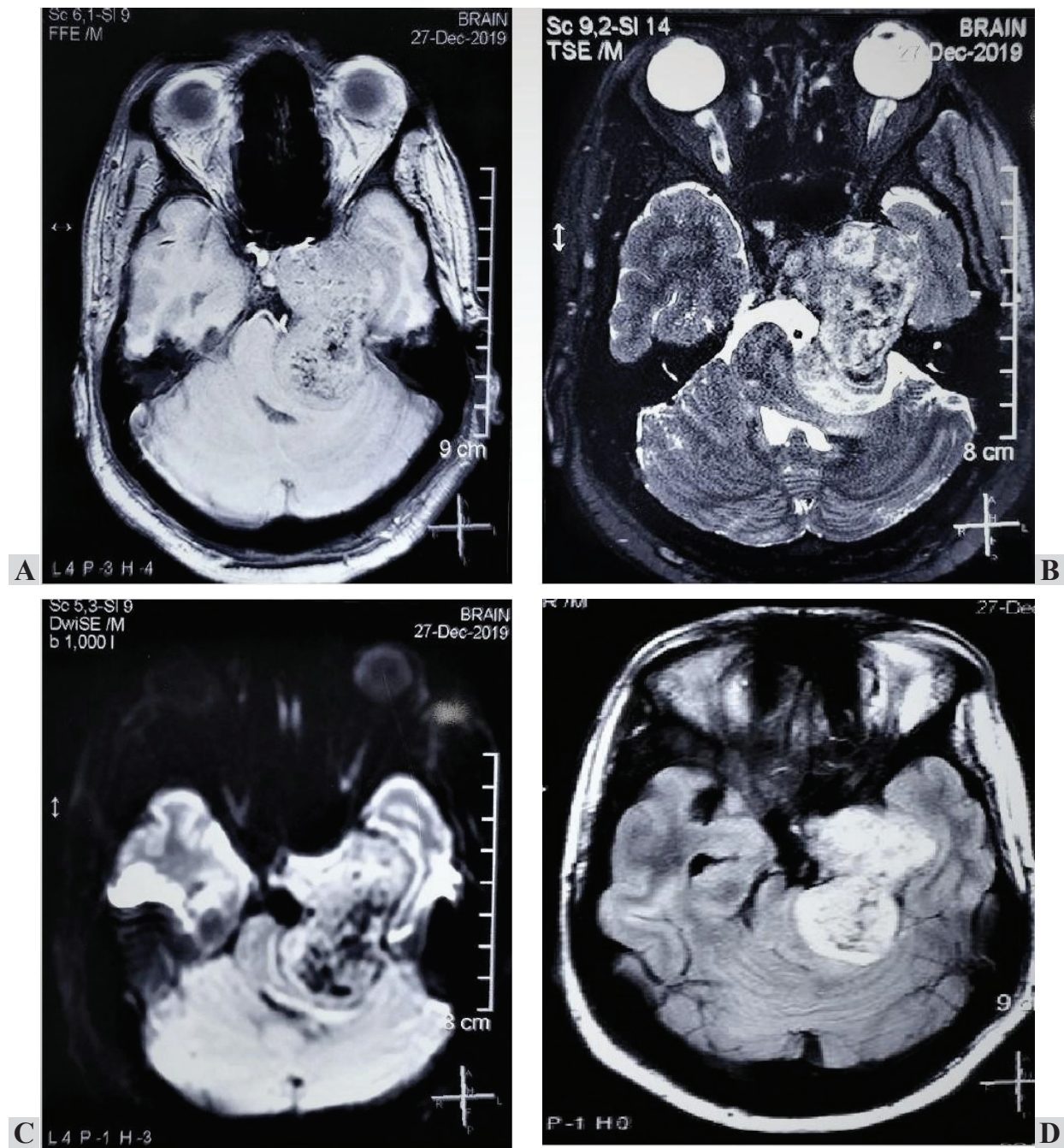

Figure 3: (A, B, D) are axial T1, T2 and FLAIR sequences respectively showing a large well marginated dumbbell shaped extra-axial T1 isointense and T2/FLAIR heterogeneously hyperintense to grey matter lesion, arising along the course of left trigeminal nerve from meckel's cave in middle cranial fossa extending to posterior cranial fossa suggestive of trigeminal schwannoma. (C) shows DWI image, the lesion is showing patchy areas of diffusion restriction.
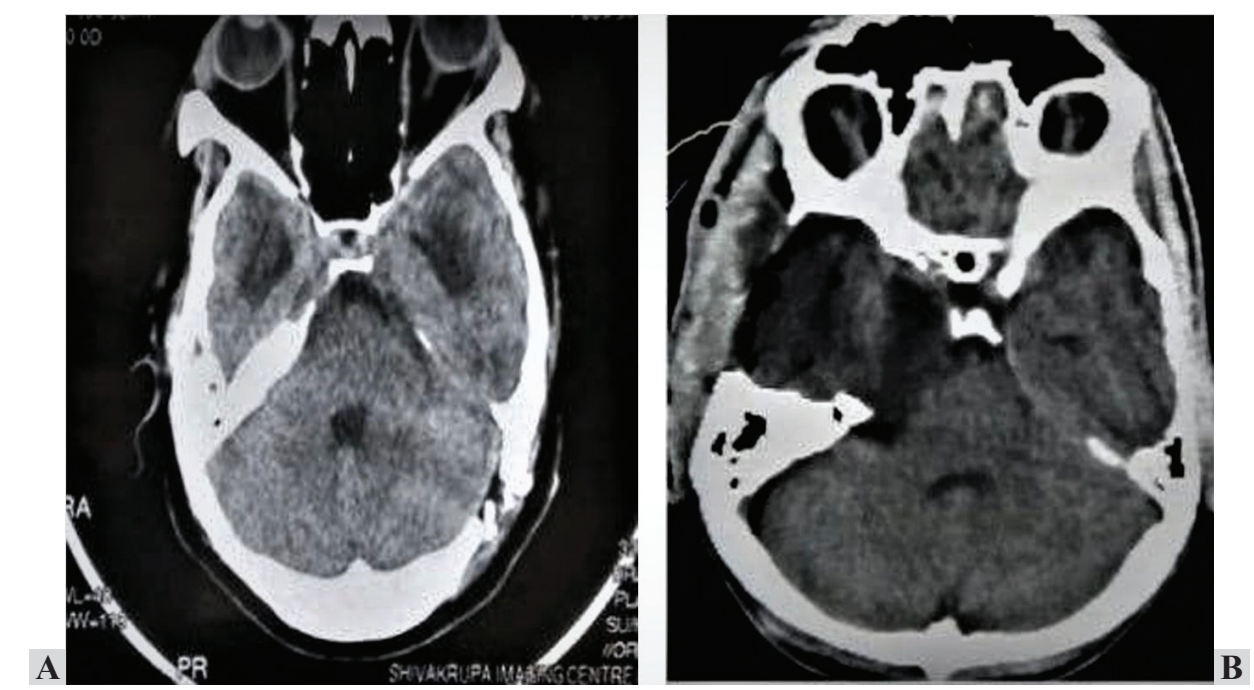

Figure 4: (A) Postoperative axial non-contrast CT showing GTR via retrosigmoid approach

(B) Postoperative axial non-contrast CT showing GTR via extended middle cranial fossa approach. 


\section{Clinical, surgical and postoperative outcome analysis of bicompartmental intracranial tumours}

\section{Discussion}

Brain tumours involving both middle and posterior cranial fossa have always been a key interest and a challenging surgery for the neurosurgeons. There is paucity of literature in regards to analysis of tumours involving both posterior and middle cranial fossa and their categorisation based on tumour types. The differential diagnoses of such tumours are trigeminal schwannoma, vestibular schwannoma, facial schwannoma, epidermoid, meningioma, ependymoma, metastasis, and chondrosarcoma..$^{1,2,3}$ Out of the 23 patients analysed retrospectively, trigeminal schwannoma (65.3\%) was the most common tumour type followed by the vestibular schwannoma (21.7\%), facial schwannoma $(8.7 \%)$ and epidermoid (4.3\%). Trigeminal schwannoma is a common tumour involving both compartments. Trigeminal schwannoma accounts for $0.8 \%$ to $8.0 \%$ of all intracranial schwannoma. ${ }^{4}$ Multicompartment trigeminal schwannoma extending to both posterior and middle cranial fossa are classified as type $\mathrm{E}$ by Ramina et $\mathrm{al}^{5}$, type MP by Yoshida and Kawase ${ }^{6}$ and type $C$ by Samii et al ${ }^{7}$. Vestibular schwannoma usually involve posterior cranial fossa (cerebellopontine angle) but can extend to middle cranial fossa.

Schwannomas are benign slow growing tumours. The lesions originate at the transition point between glial cells and Schwann cells, called as the ObersteinerRedlich zone. ${ }^{8}$ Microscopically, Schwannomas are made up entirely of neoplastic Schwann cells arranged in two distinct tissue patterns: Antoni A and Antoni B (Figure 1). Antoni A tissue is made up of compact spindle cells with elongated nuclei and ample pink cytoplasm. Verocay bodies (Figure 1), which are alternating arrangements of palisading nuclei and cell bodies, are found within Antoni A tissue. ${ }^{9}$ Epidermoid cysts consists of a connective tissue lamina lined with stratified squamous epithelium. Desquamation of epithelial cells leading to formation of pearly shiny debris, along with cholesterol and keratin secretion inside the cyst results in increase in size of the tumor. ${ }^{10}$ (Figure 1).

Preoperative imaging with computed tomography (CT) or Magnetic resonance imaging (MRI) with CT angiography has significantly improved surgical planning and outcome prediction in these tumors. ${ }^{11}$ (Figure 2, 3). Postoperative imaging with CT or MRI helps in following tumour progression.

The incidence of trigeminal schwannomas involving multiple cranial fossa varies from $27 \%$ to $59 \%$. ${ }^{12,13}$ Trigeminal schwannoma localisation to middle, posterior or multiple cranial compartments depends on its origin along the course of trigeminal nerve from gasserian ganglion, root and three divisions of $\mathrm{CN} \mathrm{V}$ respectively. ${ }^{14}$ Jefferson had classified trigeminal schwannomas occupying both middle and posterior cranial compartments as dumbbell tumors or Type C tumors. ${ }^{15}$ Day and Fukushima modified the scheme and classified trigeminal schwannoma arising from extra cranial part of $\mathrm{V} \mathrm{CN}$ as type D. ${ }^{14}$ These bicompartmental tumours are also classified as type E by Ramina et al. ${ }^{5}$ Various studies report $62 \%$ to $81 \%$ of Trigeminal Schwannomas occupying the middle and posterior cranial compartment. 16-18 Vestibular schwannoma are usually confined to posterior fossa but may invade middle cranial fossa when enlarged. There are various case reports regarding epidermoid and facial nerve schwannoma involving both middle and posterior cranial fossa. ${ }^{19-21}$. In this study, vestibular schwannomas were predominantly occupying the posterior cranial compartment with extension into the middle cranial fossa. The incidence of epidermoids extending in both posterior and middle cranial compartment ranges from $12 \%-20 \%$ of intracranial epidermoids. ${ }^{22}$ In this study only 1 case of bicompartmental epidermoid was operated.

In this study, the mean age was 46 years with a slight female preponderance $(56.5 \%)$. Trigeminal schwannomas occur predominantly in the 4th-6th decades, slightly more common in females. ${ }^{1}$ In Vestibular schwannoma, the mean age of occurrence is 58 years, and both sexes are affected equally. ${ }^{23}$ Facial schwannomas are uncommon benign tumors that can affect any age group. However, its incidence rises between the 3rd and 6th decade of life. The incidence is equal in both males and females. ${ }^{24}$ In Epidermoid, the average age at presentation is 40 years. ${ }^{25}$

Predominant presenting complaints of patients with these bicompartmental tumors were headache $(91.3 \%)$ followed by facial numbness/pain (78.3\%), ataxia (52.2\%), facial paresis/paralysis (43.5\%) and hearing loss (43.5\%). In Trigeminal schwannoma, trigeminal nerve involvement complaints are the starting symptoms in most affected patients (reported in $90 \%$ to $100 \%$ of cases). Facial hypoesthesia is the most common trigeminal symptom that usually accounts for $70 \%$ of affected patients. ${ }^{26}$ Mostly, all 3 divisions of trigeminal nerves are involved. Facial pain/ paresis is less common. Diplopia is attributed to CN IV compression by these bicompartmental tumors. ${ }^{26}$ Tumors extending to petrous bone and causing its destruction may lead to loss of hearing and CN VII dysfunction due to damage to the internal ear and internal acoustic meatus. ${ }^{27}$

Most common cranial nerve involved was $\mathrm{CN} \mathrm{V}$ (73.9\%) followed by CN VII (43.5\%) and CN VIII (26.1\%). Other cranial nerves involved were CN II, CN IV and lower cranial nerves (CN IX, X). These patterns are directly related to greater proportion of trigeminal schwannoma followed by vestibular schwannoma. Cerebellar signs were present in $52.2 \%$ of patients.

For trigeminal schwannoma we used Dolenc's frontotemporal epidural $(40 \%)$, retrosigmoid approach $(26.7 \%)$, extended middle cranial fossa $(13.3 \%)$ and 


\section{Singh et al}

combined approach (20\%). For vestibular schwannoma; retrosigmoid $(60 \%)$, extended middle cranial fossa $(20 \%)$ and combined (20\%) approaches were used. For facial schwannoma and epidermoid, retrosigmoid approach was used. Surgical approach was used on the basis of predominant fossa occupied by the tumour. For tumours having significant involvement of both posterior and middle cranial fossa, combined approach was used while predominant posterior fossa tumors were approached by retrosigmoid approach (Figure 4 A). Tumors involving predominantly middle cranial fossa were approached through an extended middle cranial fossa approach and Dolenc's approach (in trigeminal schwannoma cases) (Figure 4 B). In these cases, the primary surgical objective was to achieve a gross total resection and in cases where GTR was not feasible intraoperatively we aimed at decompression of the involved nerves and brain stem by evacuation of tumor contents and excision of nonadherent capsule, with parts of the capsule adherent to the surrounding critical structures being left behind.

Identification of involved cranial nerves at places of their exit at the skull base facilitates removal of schwannoma and epidermoid around these nerves. Surgical dissection was done along the nerves, separating it from the tumor. Electromyography (EMG) is used intraoperatively for monitoring of the cranial nerves functions, and brainstem auditory evoked potentials for monitoring cranial nerve VIII function in patients with serviceable hearing. Nine patients $(39.1 \%)$ underwent postoperative radiotherapy, all of whom received STR. The experience with fractionated radiation therapy for trigeminal schwannoma patients is very limited. Fractionated stereotactic radiotherapy may be an option for patients with large tumour who are higher risk for microsurgical resection and not a candidate for Stereotactic radiosurgery. ${ }^{28}$

New cranial nerve deficits occurred in $21.7 \%$ consisting of CN VII (17.4\%) and lower cranial nerves (4.3\%). No significant difference was found between GTR and STR group with respect to new cranial nerve deficit, systemic and regional complications. STR was significantly associated $(\mathrm{p}<0.05)$ with persisting cranial nerve deficit $(\mathrm{p}$ $<0.0343)$ and tumour progression $(\mathrm{p}<0.0001)$. At a mean follow-up duration of 9.2 months (range 0-24 months), 9 patients $(39.13 \%)$ had tumour progression. All patients with STR showed tumour progression. Subtotal tumor removal is warranted when difficult adhesions that occur between tumor and critical structures are present. Studies have shown that Greater residual tumor volume and residual disease within the internal auditory canal predicts progression. ${ }^{29}$ Midbrain compression $(\mathrm{p}=0.0343)$, internal carotid artery $(\mathrm{p}=0.0285)$ and cavernous sinus involvement $(p=0.0285)$ were also significantly associated with STR. To avoid injury to these critical structures, when GTR is not feasible, surgical decompression along with excision of nonadherent capsule is preferred.
Systemic complications were more after resection of Trigeminal schwannoma $(66.7 \%)$ followed by Vestibular schwannoma $(60 \%)$. This can be attributable to larger size and vicinity to midbrain, cavernous sinus and other critical structures. At our centre we do not prefer lumbar drain preoperatively. Meticulous dural closure in watertight fashion and occasional use of fibrin glue in cases where there is risk of CSF leak is the protocol at our centre. In this study cohort also we get only 1 case of CSF leak that resolved spontaneously on conservative management. Systemic complication peculiar to epidermoid cyst is aseptic meningitis due to spillage of cyst content intraoperatively which must be tackled carefully to avoid such complication. Irrigation with corticosteroids intraoperatively at surgical site after epidermoid resection has been recommended by few authors, ${ }^{30,31}$ and long-term postoperative corticosteroid administration is universally recommended. ${ }^{30-34}$

\section{Conclusion}

Trigeminal schwannoma is the most common tumour involving both middle cranial and posterior fossa. Most common cranial nerve involved in such tumours is $\mathrm{CN}$ $\mathrm{V}$ followed by $\mathrm{CN}$ VII. The most common presenting symptom is headache followed by facial numbness / pain. Combined approach for such tumours is required in tumours having significant size in both middle cranial fossa and posterior cranial fossa. Retrosigmoid approach is preferred for tumour mainly confined to posterior cranial fossa with small extension to middle cranial fossa. Most common new cranial nerve deficit in such tumours is CN VII. STR is significantly associated with persisting cranial nerve deficit and tumour progression. Midbrain compression, internal carotid artery and cavernous sinus involvement cause difficulty in GTR and are significantly associated with STR.

\section{Conflict of Interest: None Source(s) of support: None}

\section{References}

1. Agarwal A. Intracranial trigeminal schwannoma. Neuroradiol J. 2015 Feb;28(1):36-41.https://doi. org/10.15274/NRJ-2014-10117

2. Wiggins RH, Harnsberger HR, Salzman KL, Shelton C, Kertesz TR, Glastonbury CM. The Many Faces of Facial Nerve Schwannoma. Am J Neuroradiol. 2006 Mar; 27:694 -99. PMID: 16552018.

3. Gohil J, Rajasekar G, Shivhare P, Nair P, Abraham M. A Rare Case of an Extensive Multi-compartment Epidermoid Presenting with Pure Motor Trigeminal Neuropathy, Case Report and Review of Literature. 
J Neurosci Rural Pract. 2019 Apr-Jun; 10(2): 364-6. https://doi.org/10.4103/jnrp.jnrp_277_18

4. Youmans \& Winn neurological surgery. 7th ed. Philadelphia: Elsevier.2017. p.1293.

5. Ramina R, Aguiar HP, Tatagiba M . The surgical management of trigeminal schwannomas, in Samii's Essentials in Neurosurgery. New York, NY, Springer. 2008. pp 155-64. PMID: 28717730

6. Yoshida K, Kawase T. Trigeminal neurinomas extending into multiple fossae: Surgical methods and review of the literature. J Neurosurg. 1999; 91:20211.https://doi.org/10.3171/jns.1999.91.2.0202

7. Samii M, Migliori MM, Tatagiba $M$, et al. Surgical treatment of trigeminal schwannomas. J Neurosurg. 1995; 82:711-8.https://doi.org/10.3171/ jns.1995.82.5.0711

8. Silk PS, Lane JI, Driscoll CL. Surgical approaches to vestibular schwannomas: what the radiologist needs to know. Radiographics. 2009;29:1955-70. https:// doi.org/10.1148/rg.297095713

9. Nelson JS, Mena H, Parisi JE, et al. Principles and Practice of Neuropathology. 2nd ed 2003 Oxford University Press New York.

10. Schiefer TK, Link MJ. Epidermoids of the cerebellopontine angle: a 20-year experience. Surg Neurol. 2008;70(6):584-90.https://doi.org/10.1016/j. surneu.2007.12.021

11. Srinivas D, Somanna S, Ashwathnarayana $\mathrm{CB}$, et al. Multicompartmental trigeminal schwannomas: Management strategies and outcome. Skull Base. 2011;21:351-8.https://doi. org/10.1055/s-0031-1287683

12. Pamir MN, Peker S, Bayrakli F, Kiliç T, Ozek MM. Surgical treatment of trigeminal schwannomas. Neurosurg Rev. 2007;30(4):329-37. https://doi.org/10.1007/s10143-007-0093-5

13. Zhou LF, Mao Y, Zhang R. Surgical treatment of dumbbell-shaped neurinomas: report of an experience with 57 cases in a single hospital. Surg Neurol. 2007;68(6):594-602.https://doi. org/10.1016/j.surneu.2006.12.065

14. Day JD, Fukushima T. The surgical management of trigeminal neuromas. Neurosurgery. 1998,42(2):23340.https://doi.org/10.1097/00006123-19980200000015

15. Jefferson $G$. The trigeminal neurinomas with some remarks on malignant invasion of the gasserian ganglion. Clin Neurosurg. 1953;1:11-54.https://doi. org/10.1093/neurosurgery/1.CN_suppl_1.11

16. Chen LF, Yang Y, Yu XG, et al. Operative management of trigeminal neuromas: an analysis of a surgical experience with 55 cases. Acta Neurochir (Wien). 2014; 156(6):1105-14.https://doi.org/10.1007/ s00701-014-2051-7
17. Goel A, Muzumdar D, Raman C. Trigeminal neuroma: analysis of surgical experience with 73 cases. Neurosurgery. 2003;52(4):783-90.https://doi. org/10.1227/01.NEU.0000053365.05795.03

18. Wanibuchi M, Fukushima T, Zomordi AR, Nonaka Y, Friedman AH. Trigeminal schwannomas: skull base approaches and operative results in 105 patients. Neurosurgery. 2012;70(1, Suppl Operative);132-44. https://doi.org/10.1227/NEU.0b013e31822efb21

19. Kankane VK, Jaiswal G, Gupta TK. Epidermoid cyst in Anterior, Middle \& Extension of Posterior cranial fossa: rare Imaging with review of Literature. Romanian Neurosurgery. 2016;3:441-5. URL:https://www.journals.lapub.co.uk/index.php/ roneurosurgery/article/view/941

20. Shenoy S N, Munish G K, Raja A. Middle cranial fossa schwannoma of the facial nerve. Neurol India. 2004;52:396-7. PMID: 15472445.

21. Nishizaki T, Ikeda N, Nakano S, Sakakura T, Abiko M, Okamura T. Cerebellopontine angle facial schwannoma relapsing towards middle cranial fossa. Clinics and Practice. 2011;1:e32.https://doi. org/10.4081/cp.2011.e32

22. Lunardi P, Missori P. Transtentorial epidermoid cysts. Acta Neurochir (Wien). 1991;113(3-4):125-30. https://doi.org/10.1007/BF01403197

23. Stangerup SE, Cayé-Thomasen $P$. Epidemiology and natural history of vestibular schwannomas. Otolaryngol Clin North Am. 2012. 45:257-68. https://doi.org/10.1016/j.otc.2011.12.008

24. Rainsbury JW, Whiteside OJ, Bottrill ID. Traumatic facial nerve neuroma following mastoid surgery: A case report and literature review. J Laryngol Otol. 2007;121:601-5.https://doi.org/10.1017/ S0022215106004993

25. Akar Z, Tanriover N, Tuzgen S, et al. Surgical treatment of intracranial epidermoid tumors. Neurol Med Chir (Tokyo). 2003; 43:275-80.https://doi. org/10.2176/nmc.43.275

26. Yoshida K, Kawase T. Trigeminal neurinomas extending into multiple fossae: surgical methods and review of the literature. JNeurosurg. 1999;91 (2):20211.https://doi.org/10.3171/jns.1999.91.2.0202

27. Pollack IF, Sekhar LN, Jannetta PJ, Janecka IP. Neurilemomas of the trigeminal nerve. $J$ Neurosurg. $\quad 1989 ; 70(5): 737-45 . h t t p s: / / d o i$. org/10.3171/jns.1989.70.5.0737

28. Niranjan A, Barnett S, Anand V, Agazzi S. Multimodality Management of Trigeminal Schwannomas, J Neurol Surg B. 2016.77:371-78. https://doi.org/10.1055/s-0036-1581138

29. Breshears JD, Morshed RA, Molinaro AM, McDermott MW, Cheung SW, Theodosopoulos PV. Residual Tumor Volume and Location Predict 


\section{Singh et al}

Progression After Primary Subtotal Resection of Sporadic Vestibular Schwannomas: A Retrospective Volumetric Study. Neurosurgery. 2020 Mar 1;86 (3),410-6. PMID: 31232426

30. Schiefer TK, Link MJ. Epidermoids of the cerebellopontine angle: a 20-year experience. Surg Neurol. 2008;70(6):584-90.https://doi.org/10.1016/j. surneu.2007.12.021

31. Talacchi A, Sala F, Alessandrini F, Turazzi S, Bricolo A. Assessment and surgical management of posterior fossa epidermoid tumors: report of 28 cases. Neurosurgery. 1998;42(2):242-51.https://doi. org/10.1097/00006123-199802000-00020

32. Kobata H, Kondo A, Iwasaki K, Nishioka T. Combined hyperactive dysfunction syndrome of the cranial nerves: trigeminal neuralgia, hemifacial spasm and glossopharyngeal neuralgia-11- year experience and review. Neurosurgery. 1998;43:1351-62.https:// doi.org/10.1227/00006123-199812000-00052

33. Safavi-Abbasi S, Di Rocco F, Bambakidis N, Talley MC, Gharabaghi A, Luedemann W, et al. Has management of epidermoid tumors of the cerebellopontine angle improved? A surgical synopsis of the past and present. Skull Base. 2008;18(2):8598.https://doi.org/10.1055/s-2007-991108

34. Singh R, Prasad RS, Singh A. Evaluation of Cerebellopontine Angle Epidermoid Presenting with Cranial Nerve Deficit: A Surgical Perspective. Asian J Neurosurg. 2020 Aug 28;15(3):573-8.https://doi. org/10.4103/ajns.AJNS_226_20 